\title{
The impact of paper prototyping on card sorting: A case study
}

\author{
Karin Slegers $^{\mathrm{a}, *}$, Verónica Donoso ${ }^{\mathrm{b}}$ \\ ${ }^{a}$ CUO | Social Spaces, KU Leuven (University of Leuven)/IBBT, Parkstraat 45, Box 3605, 3000 Leuven, Belgium \\ ${ }^{\mathrm{b}}$ Institute for Media studies, KU Leuven (University of Leuven), Parkstraat 45, Box 3603, 3000 Leuven, Belgium
}

\section{A R T I C L E I N F O}

\section{Article history:}

Received 24 July 2010

Received in revised form 19 March 2012

Accepted 28 May 2012

Available online $\mathrm{xxxx}$

\section{Keywords:}

Card sorting

Methodology

Paper prototyping

Near Field Communication

User-centered design

User interfaces

\begin{abstract}
A B S T R A C T
Combining the techniques of paper prototyping and card sorting into a single session has the benefits of helping users to understand a new technology on the one hand, and of gaining insight into the users' mental models of that technology on the other hand. However, acquainting users with a new technology via a paper prototype might affect their mental models, as assessed with the card sorting technique. The aim of this paper was to explore the possibility of combining the two techniques in a single research session. Thirty-seven users participated in a study concerning a payment system based on Near Field Communication (NFC). Eight group sessions were organized, including both a paper prototyping exercise and a card sorting exercise. The order of the exercises was alternated. The findings of this case study seem to suggest that the paper prototyping exercise resulted into deeper insights into the participants' mental models resulting from the card sorting exercise. At the same time, paper prototyping seemed to prevent participants to come up with new names for their card sorting categories.
\end{abstract}

(c) 2012 British Informatics Society Limited. Published by Elsevier B.V. All rights reserved.

\section{Introduction}

User-centered design is an approach for designing products and applications based on information concerning the problems, needs, interests and capabilities of the potential users (e.g. Norman, 1988). Essential to this approach is to explicitly focus on the users in all phases of product development and to actively involve users in those phases. Common research methods in user-centered design include ethnographic methods (e.g. observation, contextual inquiry, interviews) as well as usability research methods (e.g. task analysis, cognitive walkthrough, usability testing).

Because individual user-centered design methods usually focus on a specific aspect of the users (e.g. a specific task, or specific user characteristics) is quite common in user-centered design to combine several methods and techniques. Approaches such as mixed methods design or multimethod design are considered essential to gain a complete understanding of behavior and user experience (e.g. Morse, 2010). While such approaches usually refer to combining qualitative and quantitative research methods, combinations of several qualitative methods can also be very useful to gain a better insight into the users. Although combining qualitative methods into one research project, or even into single research sessions, is quite common practice in user-centered design research, little is known about the mutual impact of the methods used. It is

\footnotetext{
This paper has been recommended for acceptance by Timothy Bickmore.

* Corresponding author. Tel.: +32 163236 52; fax: +32 16323210 .

E-mail addresses: Karin.Slegers@soc.kuleuven.be (K. Slegers), Veronica.Donoso@ soc.kuleuven.be (V. Donoso).
}

important to understand this mutual impact in order to correctly interpret the results of the research performed. In case, for instance, one method has an effect on participants' behavior, attitudes, or opinions, the results of any additional method may be affected subsequently. In this paper we present a study that included two common methods in user-centered design: card sorting and paper prototyping. Although the study was not set up to experimentally test the mutual impact of the methods used, it provides an interesting case study to explore this mutual impact.

Card sorting and paper prototyping are two qualitative techniques that are frequently applied in user-centered design and in human-computer interaction research. Both techniques are especially useful in the design of innovative applications. Such applications often involve new and unfamiliar technologies or interaction styles. To make sure that the user interface design of a new application and the interaction between users and the application are usable, it is essential to understand the users' mental models in an early stage of the design process and to actively and iteratively involve the users throughout the design process.

A method that is often used to make sure that conceptual models and user interfaces of new applications match the mental models of the end-users is card sorting (Stone et al., 2005; Brinck et al., 2002; Courage and Baxter, 2005). Card sorting allows researchers to explore the way end-users group items into categories and concepts. When performing a card sorting exercise, names of items (e.g. menu items) are printed on cards. These cards are shuffled, after which end-users are asked to group the cards into categories that they consider meaningful. Subsequently, the end-users give each of the categories a suitable name. Designers may use the 
insights gained from card sorting exercises as input for the user interface design, especially with respect to the structure of the interface menus and to terminology. In addition, card sorting provides information about which items are missing, which items were not expected by end-users, or which items should be renamed.

Since innovative applications often involve new and unfamiliar technologies or interaction styles, these are typically very difficult for end-users to understand. In order to form realistic mental models, it is essential that users thoroughly understand the new concept and the consequences of its use in their everyday lives. Moreover, it is believed that mental models consist of the user's knowledge of a system, resulting in a model that describes and predicts the system's performance as expected and perceived by the user (Jonassen, 1995). Thus, before conducting a card sorting exercise, researchers need to make sure that the users understand the potential use and impact of a new application. Or, as Rugg and McGeorge put it (Rugg and McGeorge, 1997, p. 84): "Card sorts require the respondent to know about the entities named on the cards; it is not possible to use entities unknown to the respondent".

An efficient technique to explain a new application to users in very early phases of the design process is paper prototyping. Low fidelity prototypes, such as paper prototypes, are considered an efficient way of visualizing and communicating concepts and of demonstrating and evaluating interactions without having to invest much in technical development yet (Stone et al., 2005; Jonassen, 1995; Snyder, 2003).

Typically, paper prototypes are created in the early phases of designing and developing new applications and are used as a tool to facilitate communication between designers or researchers on the one hand and end-users on the other hand (Beyer and Holtzblatt, 1998). Paper prototypes usually are sketches of the user interface design, possibly including moveable parts to represent interface elements (e.g. buttons, windows, menus) (Benyon et al., 2005). They allow the representation of action-oriented elements in an interface and of more aesthetically driven aspects (Brinck et al., 2002). As such, paper prototypes are useful to evaluate the design with users or to test the usability of the interfaces by asking users to carry out real-life tasks with the prototypes. In the latter case, the users select options or actions and the researcher shows the next interface state. Doing such tests may reveal usability problems in a very early phase of the design process and at the same time allows users to gain early hands-on experience with an interface, facilitating user experience evaluation at this stage (Reilly et al., 2005).

Combining the techniques of paper prototyping and card sorting seems a promising approach for the design of innovative applications since it allows for assessing users' mental models while making sure that the users understand the concept of the application thoroughly. As a result of using this combination of techniques, more realistic mental models may be assessed than using card sorting alone. On the other hand, a possible disadvantage of this combination of techniques is that presenting users with a paper prototype could affect their mental models. When sorting the cards, users might simply copy the model of the application that was presented to them in the form of the paper prototype, instead of forming their own mental models. In that case, the results of a card sorting exercise following a paper prototype exercise do not represent the mental models of the users, but rather those of the designers who created the prototype in the first place. Very little research has been reported showing such an influence of paper prototyping on card sorting, except for some case study descriptions (e.g. Spencer (2009) described card sorting results that were influenced by a previous usability test (p. 12)). Therefore, it is important to understand the influence of explaining new applica- tions to users via paper prototypes on the formation of users' mental models.

The aim of this paper is to explore the effect of explaining a new application to end users by means of a paper prototype on the results of a subsequent card sorting exercise involving elements from that new application. In the study described here, the order of the paper prototyping exercise and the card sorting exercise was manipulated in test sessions with end-users. By alternating this order between groups of users, the mental models of users who had been presented with a paper prototype could be compared to mental models of those users who had not.

\subsection{Context: Near Field Communication mobile payment}

The test sessions were organized within the framework of a project in which a Near Field Communication (NFC) mobile payment application was developed (Slegers, 2008a,b). NFC is a wireless communication technology that enables the exchange of data between devices over a distance of about ten centimeters (Ortiz, 2010). Increasingly more mobile phones are equipped with NFC technology, allowing for the use of the mobile phone for services such as wireless payment, ticketing, and smart posters. The application that was used in the current study runs on a mobile handset that connects to a cash terminal via NFC when the two devices touch.

The scope of the payment application is on vouchers, and in particular on so-called "meal vouchers". Meal vouchers are commonly issued in Belgium by employers to compensate for the fact that no free-of-charge meal is offered in a corporate restaurant. For both employers and employees, meal vouchers offer certain fiscal advantages. Meal vouchers typically have a value in the range of 5-10 Euros and employees receive one voucher for each working day every month. Meal vouchers can be used to pay for food in many food stores and restaurants. Each month, in combination with the meal vouchers, employees also receive a number of discount coupons for several products.

The research that was conducted for the development of this new NFC meal voucher application followed a user-centered design approach. This was done for two main reasons. The first reason was related to the fact that a completely new application was developed. Such a completely new application allows designers to ensure a close match between the users' needs on the one hand, and the functionality, usability and user experience of the application on the other hand. In addition, it provides an opportunity for creating a solution to the problems users experience with the current paper meal vouchers. The first phase of the user-centered design research aimed at assessing the users' needs and current problems and included interviews, observations and cocreation sessions. The results of this first research phase have been described in [Removed for anonymous review].

A second reason for following a user-centered design approach was the fact that most potential end-users of the new mobile payment application are still very unfamiliar with NFC-technology or with touch-based interactions. Therefore, when designing such a new application, it is important to thoroughly understand the users' mental models of such technologies and interactions. The aim of the second phase of the user-centered design research, of which the study described in this paper was a part, was to understand the potential end-users' mental models of NFC technology in general and of the concept of the NFC-enabled meal voucher in particular. For this purpose, a card sorting exercise was conducted. This information about the mental models resulting from this exercise would be used as input for the user interface design of the NFC meal voucher application.

In the first research phase of the project we learned that users had difficulties to understand the concepts of NFC technology 
and of the specific NFC meal voucher application. For instance, many participants found it hard to understand how their meal vouchers could be transferred to a shop via a mobile phone. They wondered where the vouchers would actually be stored. Also, participants who were not familiar with wireless communication were reluctant to believe that a mobile phone could really communicate with a cash terminal just by touching the two devices. Because of these difficulties, it was decided to communicate the concept to the participants in later research phases in the form of a paper prototype. This prototype served two goals. First, it allowed for the demonstration of the concept of the application on both a functional level an on the level of the look and feel of the application. Second, in addition to explaining the NFC meal voucher concept, the paper prototype could also be used to evaluate the usefulness of the concept and both usability and user experience aspects of the prototyped interfaces in a very early research phase. The paper prototype was based on the original conceptual model of the NFC meal voucher as well as on the insights that were gained about the users' needs and current problems.

The set up of the study described above was not aimed at structurally and experimentally testing the mutual impact of the two research methods used. However, the approach towards communicating a concept and assessing users' mental models in a single session allowed us to explore the combination of paper prototyping and card sorting. As a result, this paper provides a case study with preliminary insights into the mutual impact of two qualitative research methods.

\section{Methods and materials}

\subsection{Participants}

Thirty-seven Belgian meal voucher users participated in the study, 22 women and 15 men. The participants did not know each other before the study. All participants had ample experience with using mobile phones. The mean age of the participants was 39 years (ranging from 26 to 65 years). Participants received an incentive for their efforts (a gift voucher of $€ 30$ for a 2-h session).

\subsection{Material}

For the card sorting exercise, each feature and user action that is represented in the NFC meal voucher concept was printed on a card, 63 cards in total. For the paper prototyping exercise, paper mock-ups of the interfaces (both of a mobile handset and of the cash terminal) were created (see Fig. 1 for an example of a paper mock-up of the mobile handset). The Nokia 6131 (NFC-enabled) phone was used as a model for the mock-ups. Each menu and each feature from the concept that appeared on the cards was represented in the interface mock-ups and vice versa.

The paper mock-ups were designed with a sketchy look. While showing the main features of the application to the participants, such a sketchy design allowed for emphasizing that the application was far from finished, giving the participants ample opportunity to criticize and provide feedback. Or, as Buxton (2007), p. 113 puts it, "Sketches are intentionally ambiguous, and much of their value derives from their being able to be interpreted in different ways".

The mock-ups were based on the conceptual model of the NFC meal voucher, that was created after the users' needs and current problems with meal vouchers were identified in the first research phase. The mock-ups of the mobile application consisted of an "NFC meal voucher" menu, which was listed in the handset's main menu. The NFC meal voucher menu had five sub menus. The "Overview" menu provided the balance of the meal vouchers and discount coupons in possession. Also, details of individual meal vouchers and coupons could be requested (e.g. individual value,

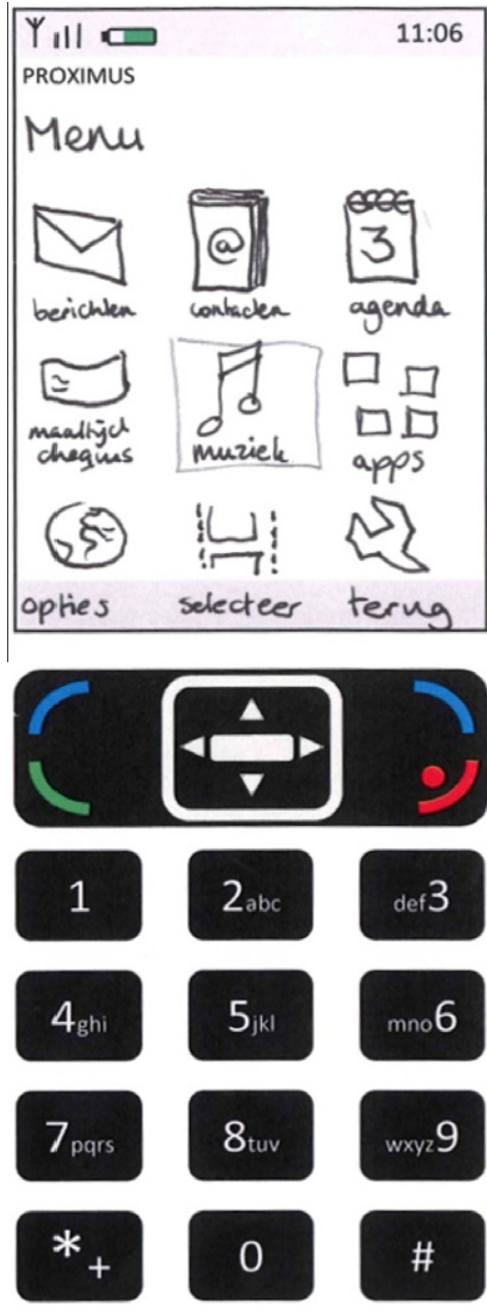

Fig. 1. Example of paper prototype mock-ups.

date of expiration, discount amount). Options to save and delete specific meal vouchers or coupons could be found in the "Options" menu, which was a submenu of the Overview menu. The "Transfer" menu allowed users to send meal vouchers or coupons to someone else, either via SMS or via NFC (by touching two handsets). A menu called "History" provided users with a chronological overview of all transactions of meal vouchers and coupons. Finally, via the "Settings" menu, users were able to set notifications in case meal vouchers or coupons would expire soon. All menus except for the History menu provided the information and options for meal vouchers and coupons separately.

\subsection{Procedure}

Four persons at a time participated in a two-hour session (in some cases only three participants showed up). Each session started with a short high-level introduction of the concept of the NFC meal voucher by means of the presentation of a storyboard. This storyboard was projected on a large screen and consisted of illustrations of a person using the NFC meal voucher. The storyboard included the main actions a user could perform with the NFC meal voucher application: receiving new meal vouchers and coupons, checking the meal voucher and coupon balance, paying with meal vouchers and coupons in a supermarket. The illustrations showed the context, the user actions, and the information on the mobile phone screen. 
After the introduction of the NFC meal voucher concept, participants had to perform two exercises: a paper prototyping exercise and a card sorting exercise. The paper prototyping exercise was done in couples to facilitate co-discovery, and therefore more natural and productive thinking aloud comments (Wilson, 1998). The couples performed the paper prototyping exercise in two separate rooms and were accompanied by a researcher. The card sorting exercise was done with the whole group, resulting in one set of sorted cards per group. The choice for doing the card sorting exercise with the whole group rather than individually was made to facilitate thinking aloud and discussions during the exercise, which is believed to yield more information about the sorting procedure (Robertson, 2002; Maurer and Warfel, 2004). In half of the sessions the participants first did the paper prototyping exercise, followed by the card sorting exercise (PP-CS condition). In the other sessions, the card sorting exercise was followed by the paper prototyping exercise (CS-PP condition).

In the paper prototyping exercise, the participants were asked to act out everyday tasks with the mock-ups (e.g. spending meal vouchers in a food store, checking the balance of their meal vouchers, viewing the transfer history). Each of these tasks was described on a single sheet of paper. The researcher manipulated the mockups so that the actions that the users performed while acting out the everyday tasks were followed by the appropriate system response. Participants were encouraged to discuss and think aloud while performing the tasks, which allowed the researchers to understand their opinions of the concept and the interfaces.

For the card sorting exercise, the cards were shuffled and put on a table in one single stack. Participants were asked to sort the cards into what they felt were meaningful categories. When they finished sorting the cards, they were asked to give an appropriate name to each category. Again, participants were stimulated to discuss and think aloud. None of the groups was specifically asked to create a menu structure for the NFC meal voucher application. Groups were allowed to leave out cards of items they did not consider important.

\subsection{Analyses}

To analyze the results of the card sorting sessions, the analysis process suggested by Spencer (2009) was followed. Photos were taken of the final categories of the card sorting sessions. Of each group, the structure (main categories, subcategories and the cards assigned for each of the categories) was subsequently recorded in an MS Excel file.

In addition to the recordings of the card sorting structures, two researchers observed the card sorting exercises and took notes of the procedure and the discussions between the participants. One researcher observed each paper prototyping session. Notes were taken of both observations and of participants' remarks and suggestions for improvements. The researchers kept track of elements in the interfaces that caused errors or problems. All notes were further analyzed by means of affinity diagramming, a method which is used to sort large amounts of data into clusters and themes (Beyer and Holtzblatt, 1998).

The structures of the PP-CS groups were compared to those of the CS-PP groups and to the structure of the original NFC meal voucher concept that was also used in the mock-ups for the paper prototyping exercise. If the paper prototyping exercise had influenced the mental models of the participants in the PP-CS groups, the structures of these groups were expected to match the structure of the original concept more closely than the structures of the CS-PP groups would, since these groups had not seen the mock-ups yet at the time they did the card sorting exercise. The differences between the structures in both conditions were explored on three levels: the content of the card sorting structures (the items that were combined in specific categories), the process of creating the card sorting structures and the naming of the card sorting categories.

\section{Results and discussion}

The results are discussed for each of the three levels of analyses mentioned above. A summary of the groups that were created by all PP-CS groups and all CS-PP groups is presented in Table 1.

\subsection{Content of the card sorting structures}

In the PP-CS condition, the four groups created ten categories that were exact copies of menus as they appeared in the paper prototyping mock-ups (the grey cells in Table 1). In the CS-PP condition, five categories were created that exactly matched one of the menus as it appeared in the original NFC meal voucher concept. When including exactly matching subcategories that were created by the groups that separated meal voucher and coupon categories, a total of nine exact matches were created.

Although the number of card sorting categories that exactly matched menus from the original NFC meal voucher concept did not differ much between the PP-CS condition and the CS-PP condition, there were some differences with respect to the content of the categories made in each condition. First of all, the categories that exactly matched menus of the original NFC meal voucher concept were not entirely the same in both conditions. In the PP-CS condition, almost all groups (except PP-CS4) copied the Transfer and the Settings menus, while this was not the case in the CS-PP condition. In this condition however, almost all groups (except for CS-PP1) created matching categories for the Overview menu. Differences that were observed for the remaining, non-matching categories related to the mock-up's menus, however, seemed small, suggesting only minor structural differences between the conditions. Most of the categories that were created during the card sorting exercise were created by groups in both conditions.

Some categories were split up into two or three subcategories or were combined with items from other menus into higher-level categories. This however did not seem to occur more often in one condition than in the other, and the splits and combinations of both conditions were quite similar in most cases. In five occasions, the PP-CS groups split items that in the original concept were combined in a single menu into two or three categories. In addition, the PP-CS groups combined items from several of the original menus into single categories twice. In the CS-PP condition such combinations occurred twice, plus three times for coupons only and once for meal vouchers only.

In summary, based on the number of matches between the card sorting categories and the original NFC meal voucher concept in both conditions, we cannot conclude that doing the paper prototyping exercise caused any differences in mental models. On the other hand, there were some differences between the two conditions with respect to the categories that were made. These differences might suggest some structural differences between the two conditions.

\subsection{Procedure of the card sorting exercise}

The most noticeable procedural difference between the two conditions that was observed was the fact that participants in the PP-CS groups had more detailed discussions about why certain cards belonged to one group and not to another. Such discussions were also observed in the CS-PP groups, but these discussions were mostly focused on the meaning and relevance of the cards.

A second procedural difference between the groups was that participants in the PP-CS groups linked the cards and the 
categories they were creating specifically to handset restrictions and menus (for instance, one participant said: "I'm trying to think of the menus of my mobile phone right now, that structure exists already, perhaps we should build on that."). Although none of the groups were specifically instructed to group the cards into categories resembling menu items, participants in PP-CS groups spontaneously referred to the menus from the paper prototyping mockup and ways to improve them (as illustrated by one of the participants: "I would find it easier if the meal voucher and coupon settings were combined into one menu, at the beginning, just as we saw in the paper handset"). In none of the groups it was observed that exact copies of menus of the original concept were deliberately made. That is, none of the groups copied a menu of the mock-up explicitly in order to replicate the mock-up's menus in the card sorting exercise. References to handset menu items were hardly observed in the CS-PP groups.

In sum, the PP-CS groups seemed to have more detailed discussions, that provided more insights into the motivations of the participants for grouping certain cards together, and thus into their mental models. In addition, the PP-CS groups appeared to think about the cards in the card sorting exercise in more concrete terms (i.e. mobile phone menus) than the CS-PP groups did, without explicitly replicating the structure of the paper prototyping exercise. That is, the card sorting exercise with the participants who had experimented with the paper prototype before was much more focused on the specific design of the concept.

\subsection{Naming of the card sorting categories}

In the PP-CS groups, names of menus as they appeared in the paper prototyping mock-up were used nine times. All groups copied the Transfer menu and also named this category 'transfer'. The Settings menu was also copied frequently, by three of the four groups (all except PP-CS4), all three copying both the content and the name of the menu in the category. Furthermore the names 'Overview' and 'History' were used once each (by PP-CS1 and PPCS2 respectively). When other names were used, often similar terms were chosen: 'admin' (PP-CS1), and 'manage' (PP-CS4) for the Options menu, 'info' (PP-CS4) for the Overview menu. In case of splitting up items into multiple categories, appropriate names were chosen that reflected the functions of the items (e.g. 'save' and 'delete' for the options to save or delete meal vouchers and coupons (PP-CS2 and PP-CS3), or 'received' and 'spent' for relevant History menu items (PP-CS1)).

In the CS-PP groups, participants were not able to copy the names of the mock-up menus because they had not seen the mock-ups yet. Nevertheless, the names 'Overview' (CS-PP2 and CS-PP3) and 'History' (CS-PP4 and CS-PP3) were used twice each. The name 'Settings' was used once in an almost identically named category called 'extra settings' (CS-PP3). Other names that were chosen often had identical meanings as the mock-up menu names (e.g. 'trading' (CS-PP1) or 'transactions' (CS-PP3) for items from the Transfer menu). As was the case in the PP-CS condition, when items from a single menu in the original concept were split up into multiple categories, appropriate names were chosen (e.g. 'sending' and 'spending' for separate subcategories of History menu items related to sending and spending meal vouchers or coupons (CS-PP2)).

To summarize, with respect to the naming of the card sorting categories it was found that in the PP-CS condition, more of the categories matching one of the mock-up menus were given identical names than in the CS-PP condition. This was not surprising, as the CS-PP groups had not seen the paper prototype mock-ups yet. Apparently, the participants who had seen the paper prototyping mock-up before doing the card sorting exercise remembered some of the menu names and used these names for the categories they created in the card sorting exercise. As was mentioned in the 
previous section, it was never observed that PP-CS groups were explicitly replicating menus from the mock-up in the card sorting exercise. Rather, the menu structure of the mock-up was redesigned to improve the structure.

\section{General discussion and conclusion}

The main goal of this paper was to explore whether the mental models of users who had been introduced to a new, unfamiliar technological application by means of a paper prototyping exercise, would differ from the mental models of users who had not done such a paper prototyping exercise first. The mental models of users were assessed by means of a card sorting exercise. Half of the users had done a paper prototyping exercise before the card sorting exercise, the other half had not. Differences between the mental models of users in both conditions were assessed on three levels: the content of the card sorting structures, the procedure of the card sorting exercise and the naming of the card sorting categories.

Although the results of this study should be interpreted with caution due to its limited scale and exploratory setup, the case study described in this paper resulted in some preliminary insights regarding the combination of the methods of card sorting and paper prototyping. First of all, regarding the content of the card sorting, the mental models of participants who had done the paper prototyping exercise differed from the models of participants who had not done this exercise. However, the mental models of the participants who did the paper prototyping exercise were not found to match the mock-ups used in the exercise more closely than the mental models of the participants who had not done the exercise. In other words, we have no reason to assume that participants simply copied the model of the application presented in the form of a paper prototype instead of forming their own mental models.

Second, on a procedural level, the paper prototyping exercise may have had a positive effect on the subsequent card sorting exercise. Moreover, gaining hands-on experience with a paper prototype before doing a group card sorting exercise lead to more discussion between participants and subsequently to more insights into the card sorting categories (e.g. motivations for grouping certain cards together) and thus into deeper insights into the participants' mental models.

Finally, the findings of this research may suggest that the paper prototyping exercise in the current study also had an effect on the level of naming of the card sorting categories. The groups who had done the paper prototyping exercise showed a larger tendency to use the menu names they remembered from the paper prototyping exercise in the card sorting exercise. Thus, it seems that familiarizing participants with the application mock-ups did not motivate them to think about names that they considered suitable for the categories.

Some aspects of the setup of the current study may have influenced the findings that were presented in this paper. For instance, in both conditions, most of the categories that were created closely matched the menu structure of the mock-up, which was only presented to the PP-CS groups. This may imply that the structure of the mock-up already closely matched the mental models of the participants of this study. Since the original NFC meal voucher concept was based on the findings of earlier user research, this is a possibility. In extreme cases, when the structure of a prototype is an exact match of users' mental models, no differences would be found between participants who have seen the prototype and those who have not seen the prototype. However, in the current study many differences were found. Although the differences concern details rather than major issues, the prototype used did not exactly match any of the mental models of the groups of partici- pants suggesting that the original concept did not already completely match the users' mental models.

A second aspect that may have influenced participants' mental models is the fact that all groups were presented with a storyboard explaining the concept of the NFC meal voucher. Even though the explanation might have had an impact on the participants' mental models, this explanation was identical for each group. As a result, the explanation itself did not have an effect on the differences between the mental models of participants in both conditions.

In addition, the fact that both the paper prototyping exercise and the card sorting exercise were done in groups rather than individually, may have led to a phenomenon called 'group think'. Although the researchers who moderated the card sorting sessions paid special attention to equal contributions to the sorting process by each of the participants in order to prevent a group think bias uncontrolled influences between participants' mental models may have occurred. It might even be argued that since mental models refer to individual psychological constructs, doing card sorting exercises in groups is not the correct approach towards assessing end-users' mental models. However, several studies (e.g. Buxton, 2007; Robertson, 2002) suggest that doing card sorting in groups leads to better insights into the sorting procedure. Since gaining such insights was one of the aims of the research phase that the current study was a part of, group card sorting exercises were preferred over individual card sorting exercises. Future research specifically aiming at studying the mutual influence of paper prototyping and card sorting may benefit from organizing individual sessions rather than group sessions.

Finally, the research described in this paper was both very exploratory and highly qualitative by nature. Although this study resulted in interesting findings with regard to combining the methods of paper prototyping and card sorting in user-centered design research, more studies should be conducted to verify these findings on a larger scale and in dedicated experimental set ups.

To conclude, the findings of this case study suggest that doing a paper prototype exercise to familiarize participants with a new technological application does not prevent participants from forming their own mental models. Moreover, doing a paper prototyping exercise before doing a card sorting exercise may result in deeper insights into the participants' mental models which is useful for (re)designing application interface structures. Therefore, we believe that combining the techniques of paper prototyping and card sorting is indeed a promising approach for the design of innovative applications. On the other hand, when the goal of a card sorting exercise is specifically aimed at improving the terminology used in an interface, doing a paper prototyping exercise may have a negative influence, as participants are less inclined to think about appropriate names for the card sorting categories.

\section{Acknowledgements}

The work described in this paper was part of the IBBT NFC Voucher project. This project was co-funded by IBBT (Interdisciplinary Institute for Technology), a research institute founded by the Flemish Government, and by IWT.

\section{References}

Norman, D.A., 1988. The design of everyday things. Basic Books, New York, NJ, USA Morse, J., 2010. Principles of Mixed Method and Multimethod Research Design. In: Tashakkori, A., Teddlie, C., Handbook of mixed methods in social \& behavioral research. Sage Publications, Inc., Thousand Oaks (CA), pp. 189-208.

Slegers, K., 2008a. Payment with Mobile (NFC) Vouchers - Using Co-Design Techniques to Identify User Requirements. Paper presented at the workshop CoDesign: Practices, Challenges and Lessons Learned at Mobile HCI, Amsterdam, The Netherlands. 2008. Available from: <http://soc.kuleuven.be/com/mediac/ cuo/?p=publication\&id=115> [retrieved 07.12 ]. 
Slegers, K., 2008b. The Use of a Lo-Fi 3D Prototype: Modeling Touch-Based Interaction in Co-Design. Paper presented at the workshop Guidelines for Haptic Lo-Fi Prototyping at NordiCHI, Lund, Sweden. Available from: <http:// soc.kuleuven.be/com/mediac/cuo/?p=publication\&id=138> [retrieved 07.12]

Stone, D., Jarrett, C., Woodroffe, M., Minocha, S., 2005. User Interface Design and Evaluation. Morgan Kaufmann Publishers, San Fransisco, CA, USA.

Brinck, T., Gergle, D., Wood, S.D., 2002. Usability for the Web: Designing Web Sites that Work. Morgan Kaufmann Publishers, San Fransisco, CA, USA

Courage, C., Baxter, K., 2005. Understanding Your Users: A Practical Guide to User Requiremtents Methods, Tools \& Techniques. Morgan Kaufmann Publishers, San Fransisco, CA, USA.

Jonassen, D.H., 1995. Operationalizing mental models: strategies for assessing mental models to support meaningful learning and design-supportive learning environments. In: Proceedings of The First International Conference on Computer Support for Collaborative Learning (CSCL), pp. 182-186.

Rugg, G., McGeorge, P., 1997. The sorting techniques: a tutorial paper on card sorts, picture sorts and item sorts. Expert Syst. 14 (2), 80-93.

Snyder, C., 2003. Paper Prototyping: The Fast and Easy Way to Design and Refine User Interfaces. Morgan Kaufmann Publishers, San Fransisco, CA, USA

Beyer, H., Holtzblatt, K., 1998. Contextual Design: Defining Customer-Centered Systems. Morgan Kaufmann Publishers, San Fransisco, CA, USA.
Benyon, D., Turner, P., Turner, S., 2005. Designing Interactive Systems: People, Activities, Contexts, Technologies. Pearson Education Limited, Harlow, UK.

Reilly, D., Dearman, D., Welsman-Dinelle, M., Inkpen, K., 2005. Evaluating early prototypes in context: trade-offs, challenges, and successes. IEEE Pervasive Computing 4 (4), 42-50.

Spencer, D., 2009. Card Sorting: Designing Usable Categories. Rosenfeld Media, New York, USA.

Ortiz, C.E., 2008. An Introduction to Near-Field Communication and the Contactless Communication API. <http://java.sun.com/developer/technicalArticles/javame/ nfc/> (retrieved 07.10).

Buxton, B., 2007. Sketching the Experience. Getting the Design Right and the Right Design. Morgan Kaufmann Publishers, San Fransisco, CA, USA.

Wilson, C., 1998. Usability Techniques: Pros and Cons of Co-participation in Usability Studies. <http://www.stcsig.org/usability/newsletter/9804-coparticipation.html> (retrieved 07.10)

Robertson, J., 2002. Information design using card sorting. <http://www. intranetjournal.com/articles/200202/pkm_02_05_02a.html (retrieved 07.10).

D. Maurer, T. Warfel, Card sorting: A definitive guide. <http://www.boxesandarrows.com/view/card_sorting_a_definitive_guide> (retrieved 07.10). 